\title{
Investigation of Substituted-Benzene Dopants for Charge Exchange Ionization of Nonpolar Compounds by Atmospheric Pressure Photoionization
}

\author{
Damon B. Robb, Derek R. Smith, and Michael W. Blades \\ Department of Chemistry, University of British Columbia, Vancouver, British Columbia, Canada
}

\begin{abstract}
Atmospheric pressure photoionization (APPI) using a dopant enables both polar and nonpolar compounds to be analyzed by LC/MS. To date, the charge exchange ionization pathway utilized for nonpolar compounds has only been efficient under restrictive conditions, mainly because the usual charge exchange reagent ions-the dopant photoions themselves-tend to be consumed in proton transfer reactions with solvent and/or dopant neutrals. This research aims to elucidate the factors affecting the reactivities of substituted-benzene dopant ions; another, overriding, objective is to discover new dopants for better implementing charge exchange ionization in reversed-phase LC/MS applications. The desirable properties for a charge exchange dopant include low reactivity of its photoions with solvent and dopant neutrals and high ionization energy (IE). Reactivity tests were performed for diverse substituted-benzene compounds, with substituents ranging from strongly electron withdrawing (EW) to strongly electron donating (ED). The results indicate that both the tendency of a dopant's photoions to be lost through proton transfer reactions and its IE depend on the electron donating/withdrawing properties of its substituent(s): ED groups decrease reactivity and IE, while EW groups increase reactivity and IE. Exceptions to the reactivity trend for dopants with ED groups occur when the substituent is itself acidic. All told, the desirable properties for a charge exchange dopant tend towards mutual exclusivity. Of the singlysubstituted benzenes tested, chloro- and bromobenzene provide the best compromise between low reactivity and high IE. Several fluoroanisoles, with counteracting EW and ED groups, may also provide improved performance relative to the established dopants. (J Am Soc Mass Spectrom 2008, 19, 955-963) () 2008 American Society for Mass Spectrometry
\end{abstract}

\begin{abstract}
$\mathrm{A}$ tmospheric pressure photoionization (APPI) using a dopant enables both polar and nonpolar compounds to be analyzed by LC/MS [1]. In this respect, APPI is unique among the established ionization methods for LC/MS, since neither electrospray ionization nor atmospheric pressure chemical ionization (APCI) are generally effective for nonpolar analytes [2]. Despite its name, analyte ionization in APPI is mostly due to ion-molecule reactions, following the photoionization of a primary reagent. The primary reagent is usually added purposefully, and is then termed a dopant, though this may not be required if a bulk component of the sample stream itself is photoionizable. Once the primary reagent ions are generated, the ensuing ion-molecule reactions may lead to analyte ionization through either proton transfer or charge exchange (electron transfer), depending upon the properties of the analyte and the chemical environment of the source. In general, polar compounds may be readily
\end{abstract}

Address reprint requests to Michael W. Blades, Department of Chemistry, University of British Columbia, 2036 Main Mall, Vancouver, B.C., Canada, V6T 1Z1, Canada. E-mail: blades@chem.ubc.ca ionizable via either ionization pathway, whereas nonpolar compounds are less amenable to proton transfer and may require charge exchange. To date, the charge exchange ionization pathway has only been efficient under restrictive conditions, mainly because of the tendency of the usual dopant ions to react by proton transfer with reversed-phase solvents and/or dopant neutrals. No study of the factors affecting the reactivity of dopant ions with solvent or dopant neutrals has previously been reported. This research is aimed at elucidating the effects of substituents on the reactivities of substituted-benzene dopant ions; another, overriding objective is to discover new APPI dopants for better implementing charge exchange ionization in reversedphase LC/MS applications.

In APPI, proton transfer ionization is commonly achieved via photoionization of toluene in the presence of methanol or acetonitrile, the usual organic solvents in reversed-phase $\mathrm{LC}$, which react with toluene photoions to produce protonated solvent reagent ions [3-5]. Acetone has also been widely used as a dopant to promote proton transfer ionization, since acetone is miscible in aqueous solvents and its photoions react with its neu- 
trals to produce protonated acetone reagent ions [6]. For charge exchange ionization, however, the reagent ions are ordinarily the dopant photoions themselves. Accordingly, for charge exchange ionization to be most effective, the dopant photoions must not be lost through reactions with the solvent, dopant neutrals, or impurities. Because of the reactivity of toluene ions with methanol and acetonitrile, the use of toluene as a dopant for charge exchange ionization of analytes delivered in these solvents is inefficient at conventional flow rates $\left(\geq 200 \mu \mathrm{L} \mathrm{min}^{-1}\right)$ and alternate approaches have been pursued. One alternative is to use only low proton affinity, normal-phase solvents such as hexane and chloroform, which are less reactive with toluene photoions $[3,4,7]$. The impressive performance of this approach was recently demonstrated in an application of APPI for the analysis of methyl esters of conjugated linoleic acid: using toluene as the dopant and $n$-hexane as the solvent $\left(600 \mu \mathrm{L} \mathrm{min}{ }^{-1}\right)$, the signal-to-noise ratio was $40 \times$ that attainable with APCI [8]. (The prospect that this kind of performance could become routinely attainable, given the right dopant, even with conventional reversed-phase LC methods, is a key motivator for the present research.) An additional option using toluene is simply to limit the flow rate of the reversedphase solvent, e.g., by adopting capillary or micro LC methods, so that reactions between the toluene photoions and the solvent are not driven to completion [9]. Another alternative is to use anisole as the dopant, since anisole photoions are stable in the presence of methanol and acetonitrile [10]. For charge exchange ionization to be effective, though, it seems the ionizing reagent must have an ionization energy (IE) well above that of the analyte [11], and anisole has a relatively low IE of 8.20 $\mathrm{eV}$, restricting its applicability (all thermodynamic data are from [12], unless otherwise indicated). Lastly, chloro- and bromobenzene appear to have great potential to serve as dopants for promoting charge exchange ionization, because these have relatively high IEs, 9.07 and $9.00 \mathrm{eV}$, respectively, and their photoions are at least partially stable under reversed-phase conditions [13]; to our knowledge, however, aside from the conference presentation of van Dam and Bruins, there have been no reports on the use of either chloro- or bromobenzene as an APPI dopant.

In the present work, diverse substituted-benzene compounds, with substituents ranging from strongly electron withdrawing (EW) to strongly electron donating (ED), were examined for their potential to serve as dopants for charge exchange ionization in reversedphase LC/MS applications. Two experimental tests were performed for each compound, using a commercial APPI source and a single-quadrupole mass spectrometer. In the first test, only the dopant candidate was introduced into the source, to isolate the dopant photoions for the second test and to evaluate their reactivity with the dopant neutrals. In the second test, reversedphase solvent (60:40 methanol/water) was added into the source, along with the dopant, to determine the reactivity of the dopant photoions with the solvent. Those compounds whose photoions are at least partially stable in the presence of both its neutrals and the solvents have the potential to be effective dopants for charge exchange ionization. Of course, to be an improvement over anisole, the best of the established dopants for charge exchange ionization in conventional reversed-phase applications, the compound must also have a relatively high IE. The results of these experiments are presented and discussed below, together with a hypothesis regarding the effects of substituents on the proton transfer reactivities of dopant photoions. Substituent effects on IEs are also described.

\section{Experimental}

\section{Chemicals}

(Trifluoromethoxy)benzene, pentafluoroanisole (97+\%), 2,4difluoroanisole, 3-fluoroanisole, 1,3,5-trimethylbenzene, and phenol were from Acros Organics (Morris Plains, NJ). Nitrobenzene, fluorobenzene, bromobenzene, anisole, and $\mathrm{N}, \mathrm{N}$-dimethylaniline were from Fluka (Buchs, Switzerland). Hexafluorobenzene, benzonitrile, (trifluoromethyl) benzene, benzaldehyde, methyl benzene carboxylate, chlorobenzene, 3-(trifluoromethyl)anisole, phenyl acetate, $\mathrm{m}$-xylene, and aniline were from Sigma-Aldrich (St. Louis, MO). Acetophenone (purity unstated) and biphenyl (purity unstated) were from Matheson Coleman and Bell (Norwood, $\mathrm{OH}$ ). Benzene was from EMD Chemicals (Norwood, $\mathrm{OH}$ ). Toluene and methanol were from Fisher Scientific (Fair Lawn, NJ). Deionized water was from an in-house generator. All chemicals were of the highest purity available, $\geq 99 \%$ unless otherwise indicated, and were used as received.

\section{Methods}

Each dopant candidate was subjected to two reactivity tests. Test 1 involved introducing neat dopant alone into the source at a flow rate of $0.2 \mu \mathrm{L} \mathrm{min}{ }^{-1}$, to isolate the dopant photoions for Test 2 and to assess their reactivity with neutral dopant. Two of the compounds, phenol and biphenyl, are solids at room temperature and were introduced as concentrated methanolic solutions: $1 \mathrm{M}$ phenol and $0.2 \mathrm{M}$ biphenyl were introduced at flow rates of 2 and $10 \mu \mathrm{L} \mathrm{min}^{-1}$, respectively, to give molar flow rates equivalent to that of neat benzene at $0.2 \mu \mathrm{L} \mathrm{min}{ }^{-1}$. A low dopant flow was used to minimize the amount of impurities added to the source, since for some compounds reactions with impurities eliminated the dopant photoions at higher flows. In Test 2, 60:40 metha$\mathrm{nol} /$ water ( $\mathrm{vol} / \mathrm{vol})$ was introduced into the source at 200 $\mu \mathrm{L} \min ^{-1}$, while the dopant flow was the same as in Test 1 , to determine the reactivity of the dopant photoions with the solvent under typical reversed-phase conditions. A dopant candidate was considered to have passed a test if the relative intensity of its photoions was substantial under the test conditions. 


\section{Instrumentation}

The APPI source was a first-generation PhotoSpray source from MDS SCIEX (Concord, ON, Canada), designed for their API 100/300/3000 series of mass spectrometers [1]. The source was powered by a custom HV supply (Electrical Services Shop, Chemistry Department, UBC) and the lamp current was set to $0.8 \mathrm{~mA}$. The offset voltage applied to the source was $1.3 \mathrm{kV}$. The heated nebulizer temperature was $400{ }^{\circ} \mathrm{C}$. The auxiliary and lamp gas flow rates were each $1.0 \mathrm{slpm}$, and the nebulizer gas flow rate was $1.9 \mathrm{slpm}(70 \mathrm{psi})$. Liquid nitrogen boil-off was used for all the gases.

The mass spectrometer was a prototype singlequadrupole instrument from MDS SCIEX (circa 1995), closely related to subsequent API 100 series instruments. The housing of the photospray source was compatible with the atmosphere-vacuum interface of the MS and was directly mounted without modification. The orifice plate and focusing ring voltages were set to 10 and $50 \mathrm{~V}$, respectively, to minimize collisioninduced dissociation. For each test, the scan range was 50-250 Da, the step size was 0.1 Da, the dwell time was $2 \mathrm{~ms}$, and 10 scans were averaged.
The dopant and solvent were delivered separately via syringe pumps from Harvard Apparatus (Holliston, MA).

\section{Results and Discussion}

Table 1 provides thermodynamic data and a summary of the experimental results for 25 substituted-benzene dopant compounds, separated into two groups. The compounds in each group are listed in order of decreasing IE. The main group has diverse substituents, ranging from strongly EW (top) to strongly ED (bottom), while the smaller groups are all fluoro-substituted anisoles. The results presented under the headings of Test 1 and Test 2 are the relative peak intensities for key ions in the test spectra; these values indicate the extent to which dopant photoions were consumed to yield the products of reactions 1-3 (see below) under the test conditions.

\section{Results for Substituted-Benzenes}

In each of the cases where dopant photoions were consumed, for the main group of compounds, one or

Table 1. Thermodynamic data and experimental results

\begin{tabular}{|c|c|c|c|c|c|c|c|c|c|}
\hline \multirow[b]{2}{*}{ Compound } & \multirow[b]{2}{*}{ Substituent(s) } & \multirow{2}{*}{$\begin{array}{l}\text { MW } \\
\text { (Da) }\end{array}$} & \multirow{2}{*}{$\begin{array}{c}\mathrm{IE} \\
(\mathrm{eV})^{\mathrm{a}, \mathrm{b}}\end{array}$} & \multirow{2}{*}{$\begin{array}{l}\mathrm{GB}(\mathrm{kJ} \\
\left.\mathrm{mol}^{-1}\right)\end{array}$} & \multicolumn{2}{|c|}{ Test $1^{\mathrm{d}, \mathrm{e}}$} & \multicolumn{3}{|c|}{ Test $2^{f, g}$} \\
\hline & & & & & $\mathrm{D}^{+\bullet}$ & $\mathrm{DH}^{+}$ & $\mathrm{D}^{+\bullet}$ & $\mathrm{S}_{2} \mathrm{H}^{+}$ & $\mathrm{P}^{+\bullet}$ \\
\hline Nitrobenzene & $\mathrm{NO}_{2}$ & 123 & 9.94 & 770 & 100 & 93 & $<1$ & 100 & n.o. \\
\hline Hexafluorobenzene & $F(\times 6)$ & 186 & 9.90 & 624 & 100 & $<1$ & $<1$ & 39 & 100 \\
\hline Benzonitrile & $\mathrm{CN}$ & 103 & 9.73 & 781 & $<1$ & 100 & - & - & - \\
\hline (Trifluoromethyl)benzene & $\mathrm{CF}_{3}$ & 146 & 9.69 & n.a. & 100 & 3 & $<1$ & 100 & n.o. \\
\hline Benzaldehyde & $\mathrm{CHO}$ & 106 & 9.50 & 802 & 1 & 67 & - & - & - \\
\hline Methyl benzene carboxylate & $\mathrm{COOCH}_{3}$ & 136 & 9.32 & 820 & 2 & 100 & - & - & - \\
\hline Acetophenone & $\mathrm{COCH}_{3}$ & 120 & 9.28 & 829 & $<1$ & 100 & - & - & - \\
\hline Benzene & $\mathrm{H}$ & 78 & 9.24 & 725 & 100 & 2 & 2 & 100 & n.o. \\
\hline Fluorobenzene & $\mathrm{F}$ & 96 & 9.20 & 727 & 100 & 6 & $<1$ & 20 & 100 \\
\hline Chlorobenzene & $\mathrm{Cl}$ & 113 & 9.07 & 725 & 100 & 3 & $46^{\mathrm{h}}$ & 100 & 69 \\
\hline Bromobenzene & $\mathrm{Br}$ & 157 & 9.00 & 726 & 100 & 10 & $31^{\mathrm{h}}$ & 100 & 22 \\
\hline Toluene & $\mathrm{CH}_{3}$ & 92 & 8.83 & 756 & 100 & 5 & $<1$ & 100 & n.o. \\
\hline Phenyl acetate & $\mathrm{OCOCH}_{3}$ & 136 & 8.60 & n.a. & $<1$ & 100 & - & - & - \\
\hline $\mathrm{m}$-Xylene & $\mathrm{CH}_{3}(\times 2)$ & 106 & 8.55 & 786 & 100 & 10 & $<1$ & 100 & n.o. \\
\hline Phenol & $\mathrm{OH}$ & 94 & 8.49 & 786 & 100 & 9 & $<1$ & 100 & n.o. \\
\hline 1,3,5-Trimethylbenzene & $\mathrm{CH}_{3}(\times 3)$ & 120 & 8.40 & 809 & 100 & 43 & 100 & 43 & n.o. \\
\hline Anisole & $\mathrm{OCH}_{3}$ & 108 & 8.20 & 807 & 100 & 25 & 100 & 7 & n.o. \\
\hline Biphenyl & $\mathrm{C}_{6} \mathrm{H}_{5}$ & 154 & 8.16 & 783 & 100 & 48 & 100 & 3 & n.o. \\
\hline Aniline & $\mathrm{NH}_{2}$ & 93 & 7.72 & 851 & 100 & 21 & 100 & $<1$ & n.o. \\
\hline $\mathrm{N}, \mathrm{N}$-dimethylaniline & $\mathrm{N}\left(\mathrm{CH}_{3}\right)_{2}$ & 121 & 7.12 & 909 & 100 & 28 & 100 & $<1$ & n.o. \\
\hline Pentafluoroanisole & $\mathrm{F}(\times 5), \mathrm{OCH}_{3}$ & 198 & 9.10 & n.a. & $<1$ & $<1$ & - & - & - \\
\hline (Trifluoromethoxy)benzene & $\mathrm{OCF}_{3}$ & 162 & $9.1^{\mathrm{c}}$ & n.a. & 100 & 9 & 39 & 39 & n.o. \\
\hline 3-(Trifluoromethyl)anisole & $\mathrm{CF}_{3}, \mathrm{OCH}_{3}$ & 176 & n.a. & n.a. & 100 & 10 & 100 & 20 & n.o. \\
\hline 2,4-Difluoroanisole & $\mathrm{F}(\times 2), \mathrm{OCH}_{3}$ & 144 & n.a. & n.a. & 100 & 12 & 100 & 6 & n.o. \\
\hline 3-Fluoroanisole & $\mathrm{F}, \mathrm{OCH}_{3}$ & 126 & 8.40 & n.a. & 90 & 2 & 75 & 1 & n.o. \\
\hline
\end{tabular}

${ }^{a}$ All ionization energy (IE) and gas-phase basicity (GB) data are from reference [12], unless otherwise indicated.

${ }^{\mathrm{b}}$ n.a. means data not available.

CAdiabatic IE estimated from date in reference [18].

${ }^{\mathrm{d}}$ The results presented are relative peak intensities.

${ }^{e} \mathrm{DH}^{+}$intensities have been corrected for ${ }^{13} \mathrm{C}$ contribution of $\mathrm{D}^{+} \cdot$

${ }^{f}-$ means Test 2 was no longer relevant, because $\mathrm{D}^{+} \cdot$ could not be isolated in Test 1.

g"n.o." means reaction 3 not observed.

${ }^{\mathrm{h}} \mathrm{D}^{+} \cdot$ value is the sum of the intensities due to both halogen isotopes. 
two of three distinct reactions were identified as being mostly responsible. The first reaction was dopant (D) self-protonation:

$$
\mathrm{D}^{+\bullet}+\mathrm{D} \rightarrow(\mathrm{D}-\mathrm{H})^{\bullet}+\mathrm{DH}^{+}
$$

Reaction 1 was prominent in Test 1 for nitrobenzene, benzonitrile, benzaldehyde, methyl benzene carboxylate, acetophenone, and phenyl acetate. For each of these, with the exception of nitrobenzene, the ratio of $\mathrm{D}^{+\bullet} / \mathrm{DH}^{+}$in its Test 1 spectrum was very small, reflecting the large extent of the reaction; nitrobenzene was the only one of these whose photoions did not react to completion, so it passed Test 1 and was subjected to Test 2 along with the compounds that did not undergo reaction 1 . The second reaction, observed in Test 2, was solvent $(\mathrm{S})$ protonation:

$$
\mathrm{D}^{+\bullet}+\mathrm{nS} \rightarrow(\mathrm{D}-\mathrm{H})^{\bullet}+\mathrm{S}_{\mathrm{n}} \mathrm{H}^{+}
$$

Reaction 2 went to completion for nitrobenzene, (trifluoromethyl)benzene, benzene, toluene, m-xylene, and phenol. For each of these, the strong $\mathrm{D}^{+\bullet}$ signal observed in Test 1 was absent in Test 2, having been replaced largely by a peak due to the protonated methanol dimer, $\mathrm{S}_{2} \mathrm{H}^{+}$. The third reaction, also observed in Test 2, appears to have been substitution of one or more methoxy groups for halogens $(X)$ on the dopant to form a product $(\mathrm{P})$ :

$$
\mathrm{D}^{+\bullet}+\mathrm{nS} \rightarrow \mathrm{P}^{+\bullet}+\mathrm{nHX}
$$

Reaction (eq 3) predominated for hexafluorobenzene and fluorobenzene. The Test 1 spectrum of hexafluorobenzene had $\mathrm{D}^{+} \cdot$ as the base peak at $m / z 186$, while its Test 2 spectrum had $\mathrm{P}^{+} \bullet$ as the base peak at $m / z 210$, but no $\mathrm{D}^{+} \cdot$. The difference in $m / z$ between $\mathrm{P}^{+} \cdot \mathrm{a}^{+} \boldsymbol{}$ is consistent with two methoxy groups having been substituted for fluorines on $\mathrm{D}^{+} \cdot$, and $\mathrm{P}$ is presumed to be dimethoxytetrafluorobenzene. Similarly, the base peak of the Test 2 spectrum for fluorobenzene was at $\mathrm{m} / \mathrm{z} 108$, presumably due to methoxybenzene, as noted previously [13], with no sign of $\mathrm{D}^{+} \cdot$. Altogether, one or more of reactions 1-3 severely the depleted the photoions of 13 of the 20 compounds in the main group of Table 1, indicating that they are poorly suited to be charge exchange dopants under conventional reversed-phase conditions.

Figures 1a and $\mathrm{b}$ are the Test 2 spectra of chlorobenzene and bromobenzene, respectively, and substantial peaks from $\mathrm{D}^{+}$are evident in each of them. Significantly, of the compounds to yield abundant $\mathrm{D}^{+}$ions in both tests, these two have the highest IEs. Thus, of all the dopant candidates examined to date, these have the potential to promote charge exchange ionization under reversed-phase conditions for the greatest number of compounds. Their spectra suggest that they are imperfect for this role, however, because their photoions do react partially with the solvent: in each spectrum, the
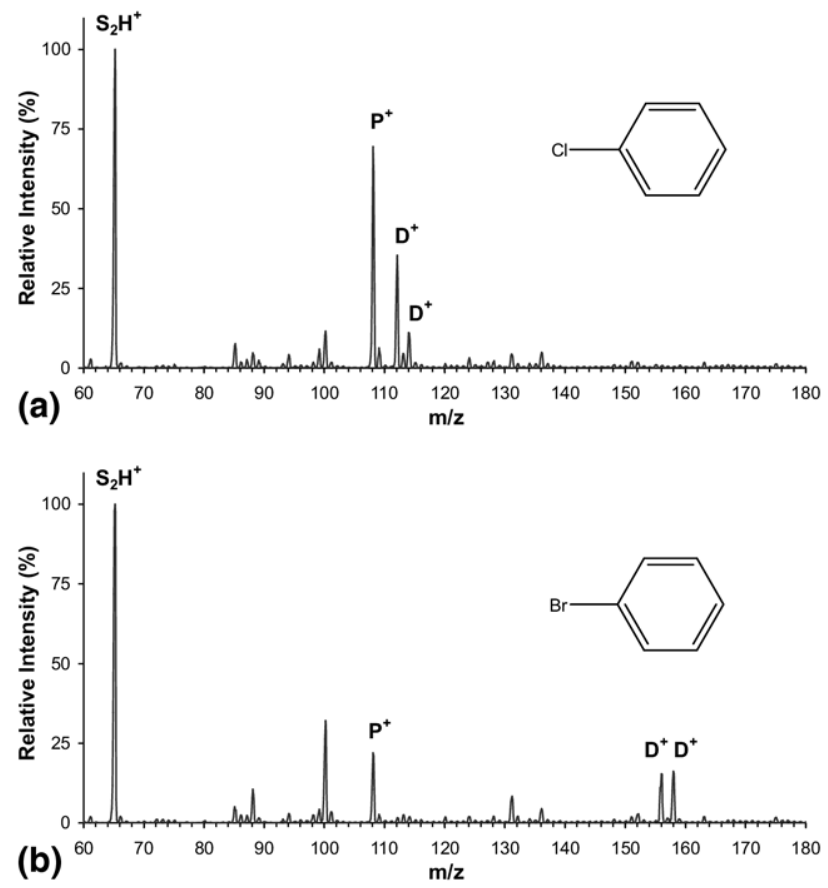

Figure 1. Test 2 spectra of (a) chlorobenzene and (b) bromobenzene. Both spectra show the dopant photoion isotopes, $\mathrm{D}^{+} \cdot$, as well as the products of reactions 2 and 3, the protonated methanol solvent dimer, $\mathrm{S}_{2} \mathrm{H}^{+}$, and the substitution product, $\mathrm{P}^{+} \cdot$ (likely methoxybenzene ${ }^{+}$), respectively.

base peak is due to $\mathrm{S}_{2} \mathrm{H}^{+}$, the product of reaction 2, and there is also a substantial peak at $\mathrm{m} / \mathrm{z} 108$, again presumably due to methoxybenzene, the product of reaction 3. Reactions 2 and 3 then both diminish the population of dopant photoions which would otherwise be available for charge exchange ionization of analytes.

Besides chloro- and bromobenzene, 1,3,5trimethylbenzene is the only compound in the first group of Table 1 to pass both tests and to have an IE $(8.40 \mathrm{eV})$ above that of anisole. In this case, the base peak of the Test 2 spectrum is due to $\mathrm{D}^{+}{ }^{\bullet}$, indicating that the dopant photoions are quite stable under the test conditions, though the substantial solvent ion peak indicates that reaction 2 does occur to an extent. 1,3,5Trimethylbenzene is then another dopant candidate which has the potential to promote charge exchange ionization under reversed-phase conditions, and its IE suggests that it, too, would be effective for a greater number of analytes than anisole.

The remaining compounds in the first group of Table 1 , anisole, biphenyl, aniline, and $N, N$-dimethylaniline, have low IEs that preclude them from being considered as general-purpose charge exchange dopant candidates. They have been included in the study to verify a hypothesized effect of ED substituents on dopant ion reactivity (see below). All of these compounds yielded photoions that were essentially unreactive under the conditions of the tests. (Their large apparent $\mathrm{DH}^{+}$ intensities in Test 1 are artifacts of contributions from 
the ${ }^{13} \mathrm{C}$ peaks of $\mathrm{D}^{+}$, which could not be accurately corrected for because the main $\mathrm{D}^{+}$peaks saturated the detector).

\section{Hypothesis Regarding Substituent Effects on Proton Transfer Reactivity}

Over the course of this research, a hypothesis was developed regarding the effects of substituents on the proton transfer reactivity of substituted-benzene photoions: EW substituents increase the tendency of substituted-benzene photoions to donate a proton to dopant or solvent neutrals, i.e., to undergo reaction 1 or 2, whereas ED substituents have the opposite, stabilizing effect, unless the ED substituent is itself acidic. This hypothesis is able to account, qualitatively, for the main experimental results, and it has significant predictive power, which eventually enabled dopant candidates to be selected rationally. It is also consistent with the findings of a pertinent study in which EW substituents were shown to increase the ring carbon acidities of substituted-benzene neutrals [14]. The theoretical basis for the hypothesis derives from the mechanism for deprotonation of unsubstituted benzene photoions, considered below.

Under the experimental conditions, benzene photoions are deprotonated by methanol, yielding phenyl radicals and protonated methanol clusters, in accordance with reaction 2, above. Implicit in reaction 2 - how it is written, when $n \geq 2$-is an intermediate step in which multiple solvent molecules bind to $\mathrm{D}^{+} \cdot$ to form an ion-solvent cluster $[3,5]$. For benzene ${ }^{+}$to be deprotonated by methanol, this step is essential because the gas-phase basicity (GB) of the phenyl radical (852 kJ $\mathrm{mol}^{-1}$ ) is greater than that of a single methanol neutral $\left(725 \mathrm{~kJ} \mathrm{~mol}^{-1}\right)$ and the reaction would be endothermic without the energy release accompanying the solvation of the proton (for simplicity, the GB values used throughout are for $\mathrm{T}=298 \mathrm{~K}$, even though the actual experimental temperatures were higher and GB contains a temperature-dependent entropy term. Estimations of $\Delta \mathrm{G}^{\circ}$ for the proton transfer reaction are little affected by this approximation, however, because the entropy terms are small and tend to cancel). For reaction 2 , the expression for the change in free-energy, $\Delta \mathrm{G}_{2}^{\mathrm{o}}$, is given by

$$
\Delta \mathrm{G}_{2}^{\mathrm{o}}=\mathrm{GB}\left[(\mathrm{D}-\mathrm{H})^{\bullet}\right]-\mathrm{GB}(\mathrm{S})+\Delta \mathrm{G}_{0, \mathrm{n}-1}^{\mathrm{o}}\left(\mathrm{SH}^{+}\right)
$$

where $\Delta \mathrm{G}_{0, \mathrm{n}-1}^{\mathrm{o}}$ is the free-energy change of the cluster formation process [15]:

$$
\mathrm{SH}^{+}+(\mathrm{n}-1) \mathrm{S} \rightarrow \mathrm{S}_{\mathrm{n}} \mathrm{H}^{+}
$$

Equation 4 clearly indicates that the solvation energy, $\Delta \mathrm{G}_{0, \mathrm{n}-1}^{\mathrm{d}}$, must drive the reaction when $\mathrm{GB}[(\mathrm{D}-$ $\left.H)^{\circ}\right]$ is greater than $G B(S)$. In addition, it reveals another important point: assuming kinetics are not a factor, differences in proton transfer reactivity for different dopant photoions must be solely attributable to differences in the strengths of their conjugate-bases, $\mathrm{GB}\left[(\mathrm{D}-\mathrm{H})^{\bullet}\right]$, since the other terms in the equation are independent of the dopant.

Further insight into the deprotonation of benzene photoions, at the molecular level, can be had from a recent, in-depth study of the hydration of ionized aromatics [16]. This study provides details of the mechanism through which water, with an even lower GB (660 kJ mol ${ }^{-1}$ ) than methanol, can deprotonate benzene $^{+\bullet}$, following the formation of a $\left(\mathrm{C}_{6} \mathrm{H}_{6}{ }^{+\bullet}\right)\left(\mathrm{H}_{2} \mathrm{O}\right)_{n}$ cluster. For the discussion at hand, the important point is in the details of the initial step in the mechanism in which a first water molecule attaches to $\mathrm{C}_{6} \mathrm{H}_{6}{ }^{+}$via a $\mathrm{C}-\mathrm{H}^{\delta+} \ldots \mathrm{O}^{\delta-}$ bond (see Figure 4 in reference [16]). Note that the positive charge on $\mathrm{C}_{6} \mathrm{H}_{6}{ }^{+}$is distributed among its hydrogens, even though the missing electron is from a ring $\pi$-orbital. This is indicative of the fact that the removal of an electron from a $\pi$-orbital must cause electron density within adjacent $\mathrm{C}-\mathrm{H} \sigma$ bonds to retract towards the ring carbons, away from the hydrogens, because electron withdrawal from the ring partially de-shields the remaining electron density from the ring's nuclear charge, causing a general contraction of all nearby orbitals. As for the $\mathrm{C}-\mathrm{H}$ bonds, these will be weakened by the retraction of electron density towards the ring, and hence the molecule's conjugate-base strength will be lowered. It stands to reason that the opposite will also be true, i.e., adding electron density to the ring must shield the existing electron density, causing an expansion of nearby orbitals, so that the ring $\mathrm{C}-\mathrm{H}$ bonds will be strengthened and the conjugate-base strength increased, absent other effects. These observations, and the previous finding regarding the significance of conjugate-base strength, $\mathrm{GB}\left[(\mathrm{D}-\mathrm{H})^{\bullet}\right]$, form the theoretical basis for our hypothesis.

We have elected not to utilize the substituent constants (s) of Hammett and Brown in our discussion, as suggested by a reviewer, because they do not themselves have explanatory power, being primarily empirically-derived constants used for predicting relative rates of particular reactions. The fact that substituent constants correlate well with molecular descriptors such as IE reflects that the same underlying electronic effects govern their values, but this does not aid in describing the nature of these electronic effects. If we were to have followed the reviewer's suggestion, our discussion would have included simply the observation that there is an imperfect correlation between reactivity in our system and substituent constants for other systems, without addressing the underlying electronic effects responsible for the correlation or the deviations from it.

\section{Substituent Effects on the Ionization Energies of Substituted-Benzenes}

For monosubstituted-benzenes, EW substituents are known to raise IE, while ED substituents lower IE 
[17-20]. A thorough analysis of substituent effects on IE and clear explanations of the effects in terms of perturbation molecular orbital (PMO) theory are provided by DiLabio et al. [20]. In brief, the EW group effect on IE is generally the result of de-shielding due to $\sigma$ inductive withdrawal, which leads to ring $\pi$-orbital stabilization (contraction), so that more energy must be supplied to remove an electron. The ED group effect on IE, on the other hand, is generally the result of filled-filled interactions between the ring $\pi$-orbitals and one or more orbitals of the substituent, which leads to ring $\pi$-orbital destabilization (expansion), so that less energy is required to remove an electron. For disubstituted benzenes, containing one EW group and one ED group, note that the effects on IE are additive. Additivity of substituent effects is also observed when there are multiple EW groups and/or weakly-ED groups; strongly-ED groups, however, may diminish or eliminate the effects of additional ED groups.

\section{Discussion of Results for Substituted-Benzenes}

Returning to the discussion of the experimental results, we first consider the dopants that undergo self-protonation, reaction 1 . Upon inspection, Table 1 reveals that all the self-protonating dopant compounds have EW substituents, with the exception of phenyl acetate, as evidenced by their elevated IEs relative to benzene. According to our hypothesis, EW substituents are supposed to increase proton transfer reactivity by lowering $\mathrm{GB}\left[(\mathrm{D}-\mathrm{H})^{\bullet}\right]$ via a weakening of the ring $\mathrm{C}-\mathrm{H}$ bonds; the result that compounds with EW groups may self-protonate is consistent with this expectation. Insight into what causes some dopants with an EW group to self-protonate while others do not can be had from Figure 2, a plot of GB versus IE for 18 of the 20 compounds in the first group of Table 1 (GB data were unavailable for phenyl acetate and (trifluoromethyl)benzene). One nota-

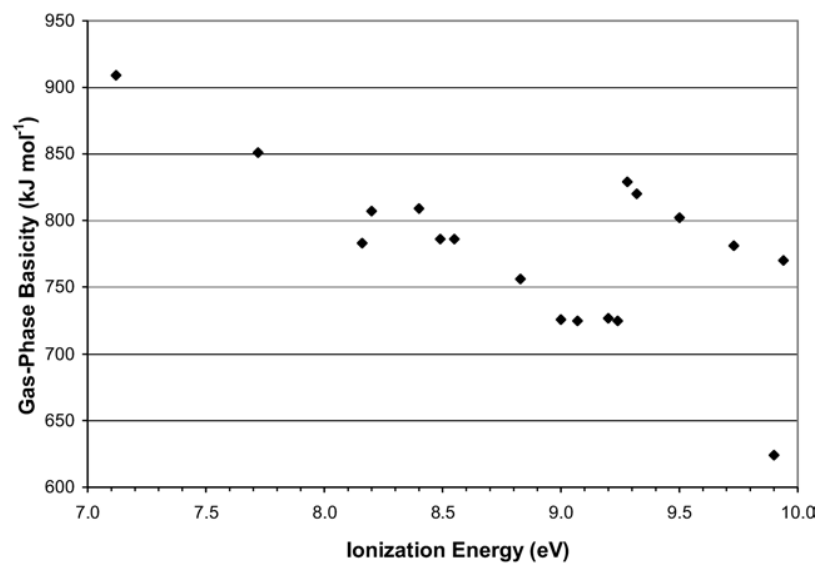

Figure 2. Plot of gas-phase basicity (GB) versus ionization energy (IE) for 18 of the 20 compounds in the first section of Table 1 (GB data were unavailable for (trifluoromethyl)benzene and phenyl acetate). The main group are ring-protonating, while the smaller group, having elevated GB values, are substituentprotonating. ble feature of Figure 2 is that it illustrates the predominant, inverse relationship that exists between GB and IE, which is due to the fact that ED substituents raise the ring's basicity and lower its IE, while EW substituents have the opposite effects, as described above. The key feature of the plot, though, is that the compounds are separated into two distinct groups, with the smaller group having GB values much higher than would be anticipated, based upon their high IEs and the clear trend in the main group. The reason for the separation is that the smaller group are substituent-protonating, rather than ring-protonating, and their GB values do not reflect the basicity of the ring, which is low because of the EW substituent effect, but that of the substituent itself [21-23]. Significantly, all of the compounds from the substituent-protonating group were observed to selfprotonate during Test 1 of the experiments; the only self-protonating dopant not among the substituentprotonating group of Figure 2 was phenyl acetate, which is absent from the plot altogether. In light of this finding and the EW substituent effect on ring carbon acidity [14], we presume then that the mechanism for dopant self-protonation involves the transfer of a proton from the acidic ring of a dopant photoion to the basic substituent of a dopant neutral, when the substituent is both EW and basic. A corollary is that selfprotonation will not be a favored reaction for compounds having an EW substituent which is weakly basic; the Test 1 result for (trifluoromethyl)benzene supports this (presuming the trifluoromethyl group is weakly basic), as might, perhaps, the Test 1 result for nitrobenzene, since it is the least basic of the substituent-protonating compounds and its photoions are the only ones from the group to be even partially stable under the test conditions. As for the anomalous case, phenyl acetate, it also most likely substituent-protonates because the acetate substituent contains a highly basic carbonyl oxygen, in addition to the carboxyl oxygen attached to the ring; however, unlike with EW substituents, we cannot be confident that the proton transferred from the photoion originates from its ring, as the ED carboxyl oxygen is believed to strengthen the ring $\mathrm{C}-\mathrm{H}$ bonds and the substituent could be more acidic than the ring, conceivably.

We now discuss the dopants whose photoions protonate the solvent via reaction 2 . Table 1 shows that reaction 2 occurs for compounds having substituents which, based upon their IEs, range from strongly EW to moderately ED. First considering the compounds with strongly EW groups, the result that nitrobenzene ${ }^{+}{ }^{\bullet}$ and (trifluoromethyl)benzene ${ }^{+\bullet}$ react to completion with the solvent is consistent with our hypothesis, since EW substituents are supposed to increase the acidity of the benzene ring. As for unsubstituted benzene itself, the mechanism through which its photoions may donate a proton to the solvent has already been addressed and will not be discussed further. The results for compounds with weakly and moderately ED substituents, however, are complicated by additional factors and require more in-depth discussion. 
Before considering the results for the compounds with weakly ED substituents, chloro- and bromobenzene, we will first address why chlorine and bromine have been classified as ED, when halogens are ordinarily considered weakly EW [24]. Here, chlorine and bromine have been classified based upon their effect on IE, as we have done throughout for all the substituents (because IE is closely related to the electronic substituent effect [25] and its value is known for most of the compounds); hence, because both chloro- and bromobenzene have slightly lower IEs than benzene, chlorine and bromine must be weakly ED. In their treatise cited previously, DiLabio et al. also classified chlorine as ED (the other halogens were not mentioned) [20], so our classification is not without precedent. As we understand it, the difference in classifications is due partly to the indirect methods ordinarily used to determine substituent effects, and partly to the ability of halogens to both donate and withdraw electron density simultaneously, through lone-pair mixing with the $\pi$ system and $\sigma$ induction, respectively. Ordinarily, substituents are classified based upon empirical determinations of their effects on the rate of electrophilic substitution elsewhere on the benzene ring [24]. In this manner, the halogens have been determined to be weakly EW overall, indicating that the $\sigma$ inductive effect predominates, slightly, under the test conditions. Note that these determinations are based upon tests involving neutral molecules, with a full complement of $\pi$ electrons to repel the halogen's lone-pairs and limit the extent to which they can be delocalized into the ring $\pi$ system. However, when a $\pi$ electron is removed by photoionization, electron-electron repulsion is reduced, enabling increased mixing between the lone pairs and the $\pi$ system. It stands to reason that the ED ability of the halogens must be enhanced when a $\pi$ electron has been removed, relative to the neutral case. We propose that this is the reason why chlorine and bromine appear to have a net ED effect in the context of IE determinations (which are also photoionization experiments), but not in the context of electrophilic substitution reactions involving neutrals. As for the Test 2 results for chloroand bromobenzene, finally, their photoions do react with the solvent via proton transfer, but to a lesser extent than benzene ${ }^{+\bullet}$. The chloro and bromo substituents then appear to strengthen the ring $\mathrm{C}-\mathrm{H}$ bonds, which is indeed what ED substituents are supposed to do. Thus, the results for chloro- and bromobenzene are consistent with our hypothesis regarding the effects of ED substituents on proton transfer reactivity, presuming chlorine and bromine are in fact weakly ED in this context.

As for the Test 2 results of toluene, m-xylene, and phenol, all having moderately ED substituents, unequivocally, the fact that their photoions react to completion with the solvent also requires further explanation, since, again, ED substituents are supposed to strengthen ring $\mathrm{C}-\mathrm{H}$ bonds and lower proton transfer reactivity. To begin, a comparison of the GBs of the benzyl (801 $\mathrm{kJ} \mathrm{mol}^{-1}$ ) and phenoxy (827 $\mathrm{kJ} \mathrm{mol}^{-1}$ ) radicals with that of the phenyl radical $\left(852 \mathrm{~kJ} \mathrm{~mol}^{-1}\right)$ confirms that toluene ${ }^{+}$and $^{\text {phenol }}{ }^{+}$are more acidic than benzene ${ }^{+\bullet}$, whereas ring-protonating toluene (756 $\left.\mathrm{kJ} \mathrm{mol}^{-1}\right)$ and phenol $\left(786 \mathrm{~kJ} \mathrm{~mol}^{-1}\right)$ are more basic than benzene $\left(725 \mathrm{~kJ} \mathrm{~mol}^{-1}\right)$. The trend in GBs for the neutral compounds, at least, is in accordance with the supposed ED substituent effect, and there is no reason to believe that the ring $\mathrm{C}-\mathrm{H}$ bonds of toluene and phenol, as well as their photoions, are not also strengthened by their ED substituents. The reason for the apparent discrepancy between the experimental results and supposition is that the substituents of toluene ${ }^{+\bullet}, \mathrm{m}^{-x y l e n e^{+\bullet}}$, and phenol $^{+}$are themselves acidic-our central hypothesis includes the caveat that ED substituents may not lower proton transfer reactivity if they are themselves acidic. Substituent acidity is partly a consequence of electron density being transferred from the substituent to the positively charged ring; i.e., for example, when a methyl substituent donates electron density to the ring, its own electron density must be diminished, thereby weakening its own $\mathrm{C}-\mathrm{H}$ bonds and increasing its acidity. It follows that the acidity of a substituent will vary in proportion to the amount of electron density donated, so that the ED substituent of an ion missing a ring $\pi$ electron will be more acidic than the same substituent of a neutral with all its $\pi$ electrons, for example. Likewise, if there are multiple ED substituents of the same type, the acidity of each substituent will be reduced as the number of substituents is increased, because the electron density donated per substituent is diminished as more are added due to increasing electron-electron repulsion about the ring. In passing, we propose that this is the mechanism responsible for the Test 2 results for 1,3,5-trimethylbenzene, whose photoions are much less reactive with the solvent than either toluene $^{+\bullet}$ or $\mathrm{m}$-xylene ${ }^{+\bullet}$, even though it also has only methyl substituents.

We now consider the dopants that apparently undergo substitution of a methoxy group for a halogen, reaction 3 . Both fluorobenzene ${ }^{+}$and hexafluorobenzene $^{+}$react to completion with the solvent under Test 2 conditions, yielding product ions consistent with methoxy groups having been substituted for fluorines. Chloro- and bromobenzene ${ }^{+\bullet}$ also appear to undergo this substitution reaction, though to a much lesser extent. We speculate that the mechanism for reaction 3 involves nucleophilic substitution, with the halogen acting as leaving group and methanol acting as nucleophile; i.e., presumably, halogens polarize the $\sigma$ bond through which they bind to the ring, via inductive electron withdrawal, leaving the ring carbons to which they are bonded slightly positive and therefore susceptible to nucleophilic attack by lone-pair electrons of methanol. However, chlorine and bromine, at least, also donate electron density into the ring $\pi$ system, through lone-pair interaction, and it is difficult to rationalize how these can be ED overall while rendering the carbons to which they are directly bonded slightly 
positive. We can only speculate that the explanation has to do with the distribution of electron density, with the donated electron density being delocalized throughout the $\pi$ system, whereas the inductively withdrawn electron density is from the single $\sigma$ bond. Fluorine, on the other hand, is less able to donate electron density into the $\pi$ system, because of its high electronegativity, so it is less difficult to conceive how it could induce a positive charge in the carbon it is bonded to. We presume then that it is fluorine's limited ability to donate offsetting electron density which makes fluorobenzene $^{+}$ undergo substitution, apparently, much more readily than chloro- and bromobenzene ${ }^{+}$. $^{\text {. }}$

Lastly, we consider the compounds whose photoions were unreactive in both Test 1 and Test 2. Anisole, aniline, and $N, N$-dimethylaniline each have strongly ED, nonacidic substituents, and the observed stability of their photoions is consistent with our hypothesis. The substituents for these compounds all donate electron density through the interaction of their heteroatom's lone-pair electrons with the ring $\pi$ system. The results for biphenyl show that its photoions are also unreactive under the test conditions. We presume that neither of biphenyl's rings would ordinarily be considered ED in a neutral molecule, because of its symmetry; however, once a $\pi$ electron has been removed by photoionization, delocalized electrons can be shared between the rings as if one were an ED substituent. Biphenyl's low IE is consistent with this explanation. Hence, biphenyl's results also appear to be in agreement with our hypothesis.

\section{Results and Discussion for Fluoroanisoles}

Finally, we examine some fluoro-substituted anisoles, compounds that we predicted during the course of our research to be likely charge exchange dopant candidates. This prediction stemmed from our central hypothesis and the idea that the electron density of anisole's ring could be adjusted to optimize the balance between low proton transfer reactivity and high IE, by adding various EW fluorines to partially offset its strongly ED methoxy group. Table 1 includes a summary of the experimental results for five fluoroanisole dopant compounds. Ionization energy data were unavailable for some of the compounds; however, we have listed them in decreasing order of their presumed IEs, based upon their number of EW fluorines and the correlation between IE and EW substituent effect. With the exception of pentafluoroanisole, all of the compounds passed Test 1 , and none of them self-protonate under the test conditions. This is as expected, since none of their substituents are basic and all of the compounds have IEs below benzene's, indicating that their rings are relatively electron rich overall and therefore presumably of low acidity. Significantly, the four fluoroanisoles to pass Test 1 all passed Test 2 as well, even though their ring electron densities have been decreased and their IEs elevated, relative to anisole. The Test 2 spectra of 2,4-difluoroanisole and 3-(trifluoromethyl)anisole were both dominated by $\mathrm{D}^{+} \cdot$ peaks, with only very small peaks due to the solvent, $\mathrm{S}_{2} \mathrm{H}^{+}$. The Test 2 spectrum for 3-fluoroanisole was similar to these two, though $\mathrm{D}^{+\bullet}$ was not the base peak because it reacted with an impurity. All three of these compounds have IEs above that of anisole, and their photoions are all very stable in the presence of solvent, so they, too, all have potential to serve as charge exchange dopants for a greater number of compounds than anisole. (Trifluoromethoxy)benzene's photoions, on the other hand, did undergo reaction 2 to a significant extent, much like chloro- and bromobenzene's, perhaps not surprisingly, considering their similar IEs [12, 18]; apparently, replacing all three hydrogens on the methoxy group with EW fluorines reduces its ED ability to the extent that it approaches that of a single chlorine or bromine substituent.

As for the only fluoroanisole to fail Test 1 , pentafluoroanisole, additional tests indicated that its photoions reacted to completion with an abundant impurity presumed to be dimethoxytetrafluorobenzene. When the concentration of pentafluoroanisole in the source was reduced below that of the usual Test 1 conditions, $\mathrm{D}^{+} \cdot$ could be isolated and subjected to Test 2, where it was found to react to completion with the solvent via reaction 3, yielding product ions again presumed to be dimethoxytetrafluorobenzene ${ }^{+}$, much like hexafluorobenzene. This result might have been expected, since $\mathrm{C}_{6} \mathrm{~F}_{5} \mathrm{OCH}_{3}{ }^{+} \cdot$ must be an intermediate in the reaction yielding $\mathrm{C}_{6} \mathrm{~F}_{4}\left(\mathrm{OCH}_{3}\right)_{2}{ }^{+}$from $\mathrm{C}_{6} \mathrm{~F}_{6}{ }^{+\bullet}$. It also indicates one of the ways in which adding excessive EW fluorines to a compound with an ED group can adversely impact its photoions' stability: if too little electron density remains in the ring, the ring carbons to which the fluorines are bonded may become sufficiently positive to attract nucleophiles. Altogether, we believe the fluoroanisole results strongly support our central hypothesis, demonstrating its predictive power.

\section{Conclusions}

This research has elucidated factors affecting the reactivity of substituted-benzene dopant photoions, and it has confirmed that chloro- and bromobenzene have substantial potential to serve as dopants for promoting charge exchange ionization in conventional reversedphase LC/MS applications. Several fluoro-substituted anisoles and 1,3,5-trimethylbenzene have also been identified as being promising charge-exchange dopant candidates. Of these dopant candidates, chloro- and bromobenzene appear to have the most immediate promise for practical use because their high IEs make them suitable for the largest number of analytes, and because they are currently available in high-purity form. All of the other promising candidates were accompanied by impurities that consumed their photoions when the dopant flow rate was increased to the levels ordinarily used in applications; additional puri- 
fication of these compounds would likely be required to optimize analytical methods using them as dopants. Since chloro- and bromobenzene both have high IEs, their photoions are largely stable in the presence of reversed-phase solvents, and they are available in highpurity form, they satisfy the basic criteria for being general-purpose charge-exchange ionization dopants. Thus, there appears to be little to gain now in pursuing further research into discovering novel dopants. The next step in this line of research is to develop and evaluate methods incorporating these new dopants for wide-ranging applications, to assess the scope of their utility and to determine what practical advantages their use may provide.

\section{Acknowledgments}

The authors gratefully acknowledge funding from the Natural Sciences and Engineering Research Council of Canada and the University of British Columbia. They also thank Peter Jacobs of NV Organon (Oss, The Netherlands) for the kind donation of the PhotoSpray source.

\section{References}

1. Robb, D. B.; Covey, T. R.; Bruins, A. P. Atmospheric Pressure Photoionization: An Ionization Method for Liquid Chromatography-Mass Spectrometry. Anal. Chem. 2000, 72, 3653-3659.

2. Hayen, H.; Karst, U. Strategies for the Liquid Chromatographic-Mass Spectrometric Analysis of Nonpolar Compounds. J. Chromatogr. A 2003, $1000,549-565$

3. Koster, G.; Bruins, A. P. Mechanisms for Ion Formation in LC/MS by Atmospheric Pressure Photo-Ionization (APPI). Proceedings of the 49th ASMS Conference on Mass Spectrometry and Allied Topics; Chicago, IL, May, 2001.

4. Kauppila, T. J.; Kuuranne, T.; Meurer, E. C.; Eberlin, M. N.; Kotiaho, T.; Kostiainen, R. Atmospheric Pressure Photoionization Mass Spectrometry. Ionization Mechanism and the Effect of Solvent on the Ionization of Naphthalenes. Anal. Chem. 2002, 74, 5470-5479.

5. Robb, D.; Blades, M. Effects of Solvent Flow, Dopant Flow, and Lamp Current on Dopant-Assisted Atmospheric Pressure Photoionization (DA-APPI) for LC-MS. Ionization via Proton Transfer. J. Am. Soc. Mass Spectrom. 2005, 16, 1275-1290.

6. Tzeng, W. B. Wei, S. Castleman, A. W. Jr. Multiphoton Ionization of Acetone Clusters: Metastable Unimolecular Decomposition of Acetone Cluster Ions and the Influence of Solvation on Intracluster Ion-Molecule Reactions. J. Am. Chem. Soc. 1989, 111, 6035-6040.
7. Impey, G.; Kieser, B.; Alary, J.-F. The Analysis of Polycyclic Aromatic Hydrocarbons (PAHs) by LC/MS/MS using a New Atmospheric Pressure Photoionization Source. Proceedings of the 49th ASMS Conference on Mass Spectrometry and Allied Topics; Chicago, IL, May, 2001.

8. Müller, A.; Mickel, M.; Geyer, R.; Ringseis, R.; Eder, K.; Steinhart, H. Identification of Conjugated Linoleic Acid Elongation and $\beta$-Oxidation Products by Coupled Silver-Ion HPLC APPI-MS. J. Chromatogr. B 2006, 837, 147-152.

9. Robb, D.; Blades, M. Atmospheric Pressure Photoionization for Ionization of Both Polar and Nonpolar Compounds in Reversed-Phase LC/ MS. Anal. Chem. 2006, 78, 8162-8164.

10. Kauppila, T. J.; Kostiainen, R.; Bruins, A. P. Anisole, a New Dopant for Atmospheric Pressure Photoionization Mass Spectrometry of Low Proton Affinity, Low Ionization Energy Compounds. Rapid Commun. Mass Spectrom. 2004, 18, 808-815.

11. Lias, S. G.; Ausloos, P.; Horvath, Z. Charge Transfer Reactions in Alkane and Cycloalkane Systems. Estimated Ionization Potentials. Int. J. Chem. Kinet. 1976, 8, 725-739.

12. http://webbook.nist.gov/chemistry.

13. van Dam, A.; Bruins, A. P. New Dopants for Atmospheric Pressure Photoionization Under Reversed Phase Liquid Chromatography Conditions. Poster presented at 21st Montreaux Symposium; November 10-12, 2004.

14. Meot-Ner (Mautner), M.; Kafafi, S. A. Carbon Acidities of Aromatic Compounds. J. Am. Chem. Soc. 1988, 110, 6297-6303.

15. Nicol, G.; Sunner, J.; Kebarle, P. Kinetics and Thermodynamics of Protonation Reactions: $\mathrm{H}_{3} \mathrm{O}^{+}\left(\mathrm{H}_{2} \mathrm{O}\right)_{\mathrm{h}}+\mathrm{B}=\mathrm{BH}^{+}\left(\mathrm{H}_{2} \mathrm{O}\right)_{\mathrm{b}}+(\mathrm{h}-\mathrm{b}+$ 1) $\mathrm{H}_{2} \mathrm{O}$, where $\mathrm{B}$ is a Nitrogen, Oxygen, or Carbon Base. Int. J. Mass Spectrom. Ion Processes 1988, 84, 135-155.

16. Ibrahim, Y. M.; Meot-Ner (Mautner), M.; Alshraeh, E. H.; Samy El-Shall, M.; Scheiner, S. Stepwise Hydration of Ionized Aromatics. Energies, Structures of the Hydrated Benzene Cation, and the Mechanism of Deprotonation Reactions. J. Am. Chem. Soc. 2005, 127, 7053-7064.

17. Crable, G. F.; Kearns, G. L. Effect of Substituent Groups on the Ionization Potentials of Benzenes. J. Phys. Chem. 1962, 66, 436-439.

18. Baker A. D.; May, D. P.; Turner, D. W. Molecular Photoelectron Spectroscopy. Part VII. The Vertical Ionization Potentials of Benzene and Some of Its Monosubstituted and 1,4-Disubstituted Derivatives. J. Chem. Soc. B 1968, 22-34.

19. Levsen, K. Substituent Effects on Ionization Potentials. In Fundamental Aspects of Organic Mass Spectrometry, Vol. IV; Verlag Chemie: New York, 1978; p. 19-21.

20. DiLabio, G. A.; Pratt, D. A.; Wright, J. S. Theoretical Calculation of Ionization Potentials for Disubstituted Benzenes: Additivity Versus Non-Additivity of Substituent Effects. J. Org. Chem. 2000, 65, 2195-2203.

21. Lau, Y. K. Kebarle, P. Substituent Effects on the Intrinsic Basicity of Benzene: Proton Affinities of Substituted Benzenes. I. Am. Chem. Soc. 1976, 98, 7452-7453.

22. Benoit, F. M.; Harrison, A. G. Predictive Value of Proton Affinity. Ionization Energy Correlations Involving Oxygenated Molecules. J. Am. Chem. Soc. 1977, 99, 3980-3984.

23. Wincel, H.; Fokkens, R. H.; Nibbering, N. M. M. Site of Protonation of Benzonitrile: Hydrogen Interchange in the Protonated Species. J. Am. Soc. Mass Spectrom. 1990, 1, 225-232.

24. Bruice P. Y. The Effects of Substituents on Reactivity. In Organic Chemistry, 3rd ed.; Prentice Hall: Upper Saddle River, NJ, 2001; p. 636-641.

25. Novak, I.; Kovac, B. Halogens in Competition: Electronic Structure of Mixed Dihalobenzenes. J. Phys. Chem. A 2005, 109, 3318-3324. 